\title{
Synergetic heating of D-NBI ions in the vicinity of the mode conversion layer in H-D plasmas in JET with the ITER like wall.
}

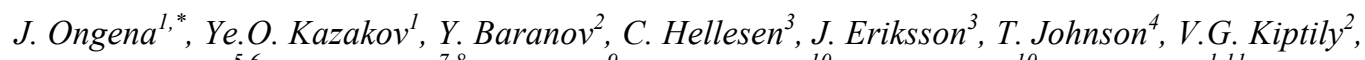 \\ M.J. Mantsinen ${ }^{5,6}$, M. Nocente ${ }^{7,8}$, R. Bilato $^{9}$, A. Cardinali $^{10}$, C. Castaldo $^{10}$, K. Crombe $^{1,11}$, A. Czarnecka $^{12}, R$. \\ Dumont $^{13}$, J. Faustin ${ }^{14}$. L. Giacomelli ${ }^{8}$, V. Goloborodko ${ }^{15}$, J. Graves ${ }^{16}$, Ph. Jacquet ${ }^{2}$, N. Krawczyk ${ }^{12}$, E. Lerche ${ }^{1,2}$, \\ L. Meneses $^{17}$, M.F.F. Nave ${ }^{17}$, H. Patten ${ }^{16}$, M. Schneider ${ }^{18}$, D. Van Eester ${ }^{1}$, H. Weisen ${ }^{17}$, J.C. Wright ${ }^{19}$ and JET \\ Contributors
}

EUROfusion Consortium, JET, Culham Science Centre, Abingdon, OX14 3DB, UK.

${ }^{1}$ Laboratory for Plasma Physics, LPP-ERM/KMS, TEC Partner, 1000 Brussels, Belgium.

${ }^{2}$ Culham Centre for Fusion Energy (CCFE), Culham Science Centre, Abingdon, OX14 3DB, UK.

${ }^{3}$ Department of Physics and Astronomy, Uppsala University, Sweden.

${ }^{4}$ KTH Royal Institute of Technology, 11428 Stockholm, Sweden.

${ }^{5}$ Barcelona Supercomputing Center (BSC), 08034 Barcelona, Spain.

${ }^{6}$ ICREA, Pg. Lluis Companys 23, 08010 Barcelona, Spain.

${ }^{7}$ Dipartimento di Fisica, Università di Milano-Bicocca, 20126, Milan, Italy.

${ }^{8}$ Istituto di Fisica del Plasma, CNR, 20125 Milan, Italy.

${ }^{9}$ Max-Planck-Institut für Plasmaphysik, 85748 Garching, Germany.

${ }^{10}$ ENEA Centro Ricerche, Frascati, Italy.

${ }^{11}$ Department of Applied Physics, Ghent University, 9000 Gent, Belgium.

${ }^{12}$ Institute of Plasma Physics and Laser Microfusion, 01-497 Warsaw, Poland.

${ }^{13}$ CEA, IRFM, 13108 Saint-Paul-Lez-Durance, France.

${ }^{14}$ Max-Planck-Institut für Plasmaphysik, 17491 Greifswald, Germany

${ }^{15}$ Institute for Nuclear Research, Kyiv 03680, Ukraine.

${ }^{16}$ EPFL, Swiss Plasma Center (SPC), 1015 Lausanne, Switzerland.

${ }^{17}$ Instituto de Plasmas e Fusão Nuclear, IST, Universidade de Lisboa, 1049-001 Lisboa, Portugal.

${ }^{18}$ ITER Organization, Route de Vignon-sur-Verdon, 13067 St Paul-lez-Durance Cedex, France.

${ }^{19}$ Massachusetts Institute of Technology, Plasma Science and Fusion Center, Cambridge, MA 02139, USA.

† See the author list of "Overview of the JET results in support of ITER" by X. Litaudon et al., Nucl. Fusion 57, 102001 (2017)

\begin{abstract}
This paper discusses the extension of the 'three-ion' species ICRF technique for heating mixture plasmas using fast injected NBI ions as resonant 'third' species. In this scenario the ICRF power is absorbed by the fast beam ions in the vicinity of the mode conversion layer where the left-hand polarized RF electric field $E_{+}$is strongly enhanced. The ions in the beam velocity distribution that have a Doppler-shifted resonance close to the mode conversion layer efficiently absorb RF power and undergo acceleration. We show first experimental observations of ICRF heating of D-NBI ions in H-D plasmas in JET with the ITER-like wall. In agreement with theoretical predictions and numerical modelling, acceleration of the D-NBI ions in this D-( $\left.\mathrm{D}_{\mathrm{NBI}}\right)-\mathrm{H}$ scenario is confirmed by several fast-ion measurements. An extension of the heating scheme discussed here is acceleration of T-NBI and D-NBI ions in D-T plasmas, offering the potential to further boost the $Q$-value in future D-T campaigns in JET.
\end{abstract}

\section{Introduction}

Plasma heating in the ion cyclotron range of frequencies (ICRF) is widely used in magnetic confinement fusion research. The fast magnetosonic waves excited in the plasma by the RF currents on the antenna strap(s) can undergo a large variety of collisionless interactions, both with ions and electrons [1]. Strong wave-particle interaction is possible when the wave frequency matches the Doppler-shifted cyclotron frequency or one of its harmonics of the plasma ions:

$$
\omega=n \omega_{c s}+k_{/ /} \mathrm{V}_{/ /}(n=0, \pm 1, \pm 2, \ldots) \text {. }
$$

Here, $\omega$ is the angular frequency of the magnetosonic wave, $\omega_{c s}=\mid q_{s} l B / m_{s}$ the local cyclotron frequency of

Corresponding author: j.ongena@,fz-juelich.de 
particle species ' $s$ ' with charge $q_{\mathrm{s}}$ and mass $m_{\mathrm{s}}, k_{\|}$is the wavenumber and $\mathrm{v}_{\|}$the particle velocity parallel to the confining magnetic field. A review of traditionally used ICRF scenarios is given in [2-4], and recent advances in physics and technology of ICRF are summarized in [5].

The electric field associated with the fast magnetosonic waves in the plasma can be written as the sum of a component rotating in the sense of the ions (left-hand polarized $E_{+}$), and one rotating in the opposite direction (right-hand polarized $E_{-}$). The RF power $P_{\text {abs }}$ absorbed by the ions at the $n^{\text {th }}$ harmonic is given by

$$
P_{a b s} \propto\left|E_{+} J_{n-1}\left(k_{\perp} \rho_{L}\right)+E_{-} J_{n+1}\left(k_{\perp} \rho_{L}\right)\right|^{2}
$$

with $\mathrm{k}_{\perp}$ the component of the wave vector perpendicular to the magnetic field, $\rho_{\mathrm{L}}$ the Larmor radius of the resonant ion and $n$ the harmonic number of the cyclotron frequency. $J_{\mathrm{m}}$ is the Bessel function of the first kind and $\mathrm{m}^{\text {th }}$ order. For fundamental heating $(n=1)$, wave absorption by thermal and moderately energetic particles (energies of a few hundred $\mathrm{keV}$ ) is to first order determined by the left-hand polarized component $E_{+}$, i.e. $P_{\text {abs }} \propto\left|E_{+}\right|^{2}$, while the contribution to power absorption due to $E_{-}$is negligibly small. Indeed, for these ions $\mathrm{k}_{\perp} \rho_{\mathrm{L}} \ll 1$ resulting for $n=1$ in $\mathrm{J}_{\mathrm{n}-1}\left(\mathrm{k}_{\perp} \rho_{\mathrm{L}}\right)=\mathrm{J}_{0}(0) \simeq 1$ and $J_{n+1}\left(k_{\perp} \rho_{L}\right)=J_{2}(0) \simeq 0$. For more energetic ions, typically in the MeV-energy range (such that $k_{\perp} \rho_{L}$ is no longer negligibly small), the situation is more complicated and both the RF-field components $E_{+}$and $E_{-}$contribute to wave absorption.

The parallel velocity needed for resonant interaction by ions with waves with an angular frequency $\omega$ follows from Eq. (1):

$$
\mathrm{v}_{\|}=\left(\omega-n \omega_{\mathrm{cs}}\right) / k_{\|}
$$

This condition can be locally fulfilled for thermal ions (low $\mathrm{v}_{\|}$) if the wave frequency closely matches with one of the ion cyclotron frequencies $\omega=n \omega_{c i}(n=1,2, \ldots)$. Larger values of the difference $\omega-n \omega_{c i}$, require higher values for $v_{\|}$. This condition alone is not sufficient for efficient power absorption by the ions. Indeed, as follows from Eq. (2), the local $E_{+}$component needs also to be sufficiently large and this is the case at the ion-ion hybrid resonance / L-cutoff pair located in between the resonance layers of the two main plasma species. In what follows we will call this pair of layers the Mode Conversion (MC) layer (see Fig. 1).

In the case of minority heating, where the concentration of one of the two ion species is typically a few percent, this MC layer is located rather close to the resonance of the minority species, and thermal minority ions can then profit from the large $E_{+}$associated with this layer to efficiently absorb power from the injected magnetosonic waves. However, if the concentration of the minority species becomes larger (typically $\sim 10-20 \%$ ), the MC layer moves radially outward, away from the minority cyclotron resonance. As a result, thermal ions can then no longer reach this layer and instead dominant electron absorption takes over.

There is an alternative way to achieve dominant ion absorption under such conditions: extend the plasma composition beyond two thermal ion species only and provide an additional population of ions that resonantly absorb RF power close to the MC layer. Two possibilities to do so follow immediately from Eq. (1). First, by adding a third ion species to the plasma with a different $Z / A$ value than that of the two main ions such that $(Z / A)_{2}<(Z / A)_{3}<(Z / A)_{1}$. Such 'three-ion' ICRF scenarios have been theoretically developed in $[6,7]$, and later confirmed in dedicated JET and Alcator C-Mod experiments [8].
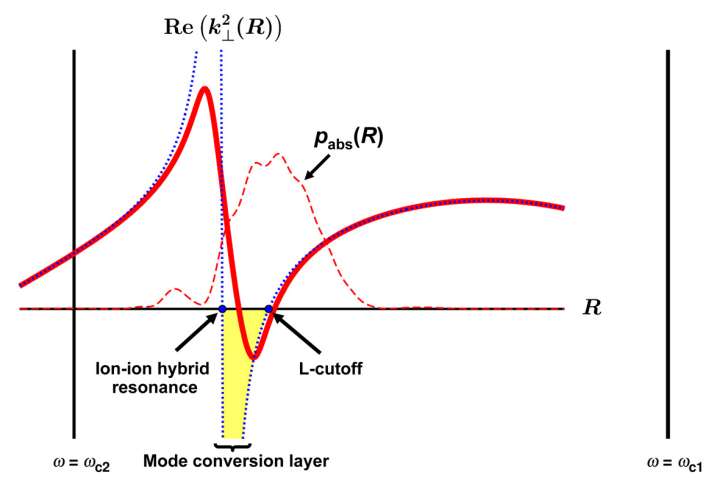

Fig. 1: Typical dispersion curve (hot-plasma approximation) for the fast magnetosonic wave in a mixture plasma, without (blue dotted line) and with a population of fast beam ions (red solid line). The position of the mode conversion layer between the ion cyclotron layers $\omega=\omega_{\mathrm{c} 1}$ and $\omega=\omega_{\mathrm{c} 2}$ can be controlled by adjusting the plasma composition. In the presence of fast ions (injected NBI ions, fusion-born alphas, etc.), efficient absorption of ICRH power occurs in the immediate neighbourhood of the mode conversion layer (red dashed line).

The second option relies on using fast ions with sufficiently large parallel velocities to make use of the Doppler-shift term $k_{\|} \mathrm{V}_{\|}$in Eq. (1). Indeed, for such fast ions the condition for resonant wave-particle interaction, Eq. (1), is fulfilled at a different radial location than for thermal ions. The radial separation between the resonant layer for the thermal ions $\omega \approx \omega_{c i}$ and that for the fast ions $\omega=\omega_{c i}+k_{\|} \mathrm{v}_{\|}$is given by

$$
\Delta R_{\mathrm{fast}} \approx n_{\mathrm{tor}} \times \mathrm{v}_{\mathrm{ll}, \mathrm{fast}} / \omega,
$$

where $n_{\text {tor }}$ is the toroidal wave number in the antenna spectrum $\left(k_{\|} \approx n_{\text {tor }} / R\right)$. In this case, the $Z / A$ value of resonant fast particles does not necessarily have to differ from that of the main plasma ions, $(Z / A)_{1}$ and $(Z / A)_{2}$. Fast ions provided by the neutral beam injection (NBI) system are a natural choice for such particles. Remark that the same physics applies to energetic ions born in various fusion reactions (e.g. ${ }^{4} \mathrm{He}$ ions from $\mathrm{D}+\mathrm{T}$ and $\mathrm{D}+{ }^{3} \mathrm{He}$ reactions). They can therefore also effectively absorb RF power in the vicinity of the MC layer in 
mixture plasmas. Absorption of ICRF power by alpha particles in D-T plasmas is well-known and has been studied earlier, see e.g. Fig. 4 in Ref. [9].

Note that the Doppler-shifted ion cyclotron absorption by D-beam ions in D plasmas was earlier reported on JET $[10,11]$. In those experiments there was no MC layer present in the plasma, and the double-pass absorption was computed to be 5-15\% [11]. Although upon crude inspection the two techniques look rather similar, the heating scenario reported here is in two aspects fundamentally different, as (i) a mixture plasma is used with a well defined plasma composition such that (ii) the fast beam ions can profit from the large $E_{+}$ located close to the MC layer resulting in a greatly enhanced double-pass absorption $(>80 \%)$.

\section{Three-ion ICRH experiments using fast beam ions as resonant particles: experimental conditions}

To test the possibility of ICRH absorption by fast beam particles in the vicinity of the MC layer, a number of dedicated pulses were performed in H-D plasmas with various values for the isotopic H/D ratio in JET with the ITER-like wall (ILW).

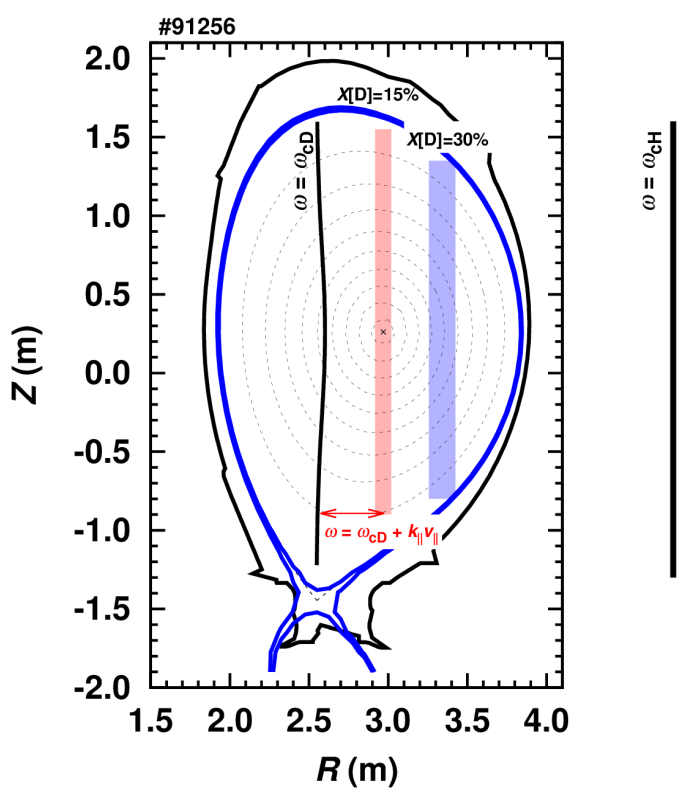

Fig. 2: Tokamak poloidal cross-section with the location of the ion cyclotron resonance layers for thermal $\mathrm{D}$ ions for JET pulse \#91256 ( $\left.B_{0} \approx 2.9 \mathrm{~T}, I_{\mathrm{p}}=2 \mathrm{MA}, f=25 \mathrm{MHz}\right)$. The computed locations of the mode conversion layer in H-D plasmas are also shown for $X[\mathrm{D}]=15 \%$ and $X[\mathrm{D}]=30 \%\left(n_{\mathrm{tor}}=27\right.$ and $X\left[{ }^{9} \mathrm{Be}\right]=0.5 \%$ assumed $)$. The reported synergetic ICRF $+\mathrm{NBI}$ scenario relies on wave absorption in the vicinity of the mode conversion layer by NBI-injected fast D ions through the Doppler-shifted fundamental ion cyclotron interaction $\omega=\omega_{\mathrm{cD}}+k_{/ /} \mathrm{V}_{\| /}$.
The operational conditions for the experiments described here were as follows: H-D plasma with $X[\mathrm{D}] \approx 10-15 \%, B_{0} \approx 2.9 \mathrm{~T}, I_{\mathrm{p}}=2 \mathrm{MA}, n_{\mathrm{e} 0} \approx 4 \times 10^{19} \mathrm{~m}^{-3}$, dipole antenna phasing at $f=25 \mathrm{MHz}$. The location of the ion cyclotron resonances for those operational parameters is shown in Fig. 2.

The JET tangential D-NBI injectors $\left(\mathrm{v}_{\mathrm{I}} / \mathrm{v} \approx 0.62\right)$ were used at an acceleration voltage of $E_{0} \approx 100 \mathrm{keV}$. This defines the maximum parallel velocity $\mathrm{v}_{\|}^{*}=2 \times 10^{6} \mathrm{~m} / \mathrm{s}$ in the slowing-down distribution of the injected beam ions, and beam particle velocities range thus from $v_{\|}=v_{\|}^{*}$ to thermal ion velocities. Taking into account that the spectrum for dipole phasing of the ICRH antennas in JET shows a maximum for the toroidal wavenumber $n_{\text {tor }}=27$ and that the fastest D-NBI ions have a characteristic Doppler shift $\Delta R_{\text {fast }} \approx 35$ $40 \mathrm{~cm}$, (Eq. (4)) we thus choose the value for the toroidal magnetic field and ICRH frequency such that the ion cyclotron resonance layer for thermal $\mathrm{D}$ ions is located at $R_{\mathrm{ic}, \mathrm{D}} \approx 2.6 \mathrm{~m}$, i.e. about $40 \mathrm{~cm}$ from the magnetic axis towards the high field side (HFS). The cyclotron resonance of $\mathrm{H}$ ions is then located at $R_{\mathrm{ic}, \mathrm{H}} \approx 5.2 \mathrm{~m}$, i.e. outside the plasma at the low field side (LFS).

H-D mixture plasmas with various isotopic compositions can be used to realize the $\mathrm{D}-\left(\mathrm{D}_{\mathrm{NBI}}\right)-\mathrm{H}$ scenario. We recall that in our experiments D concentrations can be used up that value for which the outward shifted position $R_{\mathrm{MC}}$ of the $\mathrm{MC}$ layer is 'overshooting' the Doppler shifted resonance position of the fastest beam ions in the distribution. In other words, the useful $\mathrm{D}$ concentrations in the plasma have to be such that the following condition is fulfilled:

$$
R_{\mathrm{MC}} \lesssim R_{\mathrm{ic}, \mathrm{D}}+\Delta R_{\mathrm{fast}}
$$

For every percent increase in the D concentration in the plasma, the L-cutoff and the ion-ion hybrid resonance layer shift to the LFS by about $2.8 \mathrm{~cm}$ and $2.2 \mathrm{~cm}$, respectively. The position of the MC layer in $\mathrm{H}-$ $\mathrm{D}$ plasmas can thus be approximated as $R_{\mathrm{MC}} \approx R_{\mathrm{ic}, \mathrm{D}} \times(1+X[\mathrm{D}])$. Combining this with Eq. (5) then leads immediately to the following estimate of the upper limit of the concentration for the D ions:

$$
X[\mathrm{D}] \lesssim \Delta R_{\text {fast }} / R_{\mathrm{ic}, \mathrm{D}}
$$

For the experimental parameters in the experiments reported here, this yields $X[\mathrm{D}]_{\max } \approx 15 \%$. At higher D concentrations $X[\mathrm{D}]>X[\mathrm{D}]_{\max }$ (e.g. $30 \%$ as also indicated in Fig. 2), the MC layer shifts further to the LFS and the separation between the $\omega=\omega_{\mathrm{cD}}$ and the MC layer becomes too large. Since for such conditions no beam ions have parallel velocities that allow them to resonate at the $\mathrm{MC}$ layer, electron damping through mode conversion becomes dominant. Maximizing absorption by fast NBI ions (and thus minimizing electron absorption) under those conditions is still possible, but requires higher NBI injection energies and/or ICRH operation with larger dominant $k_{\|}$. 
For the calculation of the location of the MC layer and optimal plasma conditions we have to take into account the total concentration of D ions (thermal and beam ions). For the described experiments, the fraction of fast D-NBI ions in the plasma core was ranging between $2 \%$ and $4 \%$. The presence of those ions modifies the fast wave dispersion as shown by the red solid curve in Fig. 1. The corresponding radial profile for the ICRF power absorption (red dashed line in Fig. 1) shows that RF power is absorbed by the fast ions in the vicinity of the MC layer. It is important to realize that the D-NBI injectors not only provide a seed of resonant fast ions, but also contribute to the H-D plasma ratio, via thermalized D-ions. This means that the proposed ICRF + NBI heating technique can also be applied for heating nearly pure $\mathrm{H}$ plasmas, where the necessary $\mathrm{D}$ ion concentration is provided by the thermalized fast ions from D-NBI injection.

\section{Experimental results}

Figure 3 shows the waveforms for JET pulse \#91256: (a) NBI and ICRF power, (b) the temporal evolution of the central electron temperature $T_{\mathrm{e} 0}$, (c) plasma stored energy $W_{\mathrm{p}}$, (d) the neutron rate and (e) the number of gamma-ray counts with $E_{\gamma}=3.37 \mathrm{MeV}$ from one of the channels of $\mathrm{D}+{ }^{9} \mathrm{Be}$ nuclear reactions.

The edge isotopic ratio $\mathrm{H} /(\mathrm{H}+\mathrm{D})$ in this pulse varied between 0.92 and 0.90 . At $t=7.5 \mathrm{~s}$, about $3.5 \mathrm{MW}$ of D-NBI power (obtained using two tangential PINIs) is injected into the plasma, resulting in central electron temperatures $T_{\mathrm{e} 0} \approx 2.0-2.2 \mathrm{keV}$, and plasma stored energy $W_{\mathrm{p}} \approx 1.05 \mathrm{MJ}\left(\Delta W_{\mathrm{p}} / \Delta P_{\mathrm{NBI}} \approx 0.13 \mathrm{MJ} / \mathrm{MW}\right)$. At $t=9.0 \mathrm{~s}, 2.5 \mathrm{MW}$ ICRF power (dipole phasing at $f=25 \mathrm{MHz}$ ) is coupled to the plasma.

As soon as the ICRH system is switched on, the central electron temperature increases up to $T_{\mathrm{e} 0} \approx 4.0 \mathrm{keV}$ and the plasma stored energy reaches $W_{\mathrm{p}} \approx 1.45 \mathrm{MJ}\left(\Delta W_{\mathrm{p}} / \Delta P_{\text {ICRH }} \approx 0.15 \mathrm{MJ} / \mathrm{MW}\right)$. While the increase in plasma stored energy per MW of injected NBI or ICRF power is similar, the ratio $\Delta T_{\mathrm{e} 0} / \Delta P_{\text {aux }}$ is significantly higher for the ICRH +NBI heating phase, namely $0.64 \mathrm{keV} / \mathrm{MW}$ vs. $0.27 \mathrm{keV} / \mathrm{MW}$.

Figure 4 shows the electron temperature profile during various heating phases of the pulse and shows clearly the difference between the $T_{\mathrm{e}}$ profiles obtained during application of NBI-only (rather broad power deposition) and during the combined ICRH $+\mathrm{NBI}$ heating phase. It clearly shows that the ICRF scenario discussed here provides a rather localized core plasma heating.

The stabilization of the sawtooth oscillations is a first clear indication for the presence of fast particles due to synergetic ICRH+NBI acceleration: from sawteeth with periods $T_{\text {saw }} \approx 0.11 \mathrm{~s}$ during the NBI-only heating phase of the pulse $(t=7.5-9.0 \mathrm{~s})$ to $T_{\text {saw }} \approx 0.53 \mathrm{~s}$ during the combined $2.5 \mathrm{MW}$ ICRH $+3.5 \mathrm{MW}$ NBI heating phase $(t=9.0-11.5 \mathrm{~s})$. Further confirmation of synergetic ICRF+NBI acceleration of the D-beam ions follows from the large increase in the neutron yield (panel (d) in Fig. 3) and from measurements of gamma rays with energy $E_{\gamma}=3.37 \mathrm{MeV}$, resulting from nuclear reactions between ${ }^{9} \mathrm{Be}$ nuclei and D particles (panel (e) in Fig. 3). The minimal fast $\mathrm{D}$ energy needed to observe gamma rays from the ${ }^{9} \mathrm{Be}+\mathrm{D}$ reaction is $\sim 500 \mathrm{keV}$ [12]. The modulation in both signals is due to the expulsion of the fast particles from the centre associated with the sawtooth crashes.

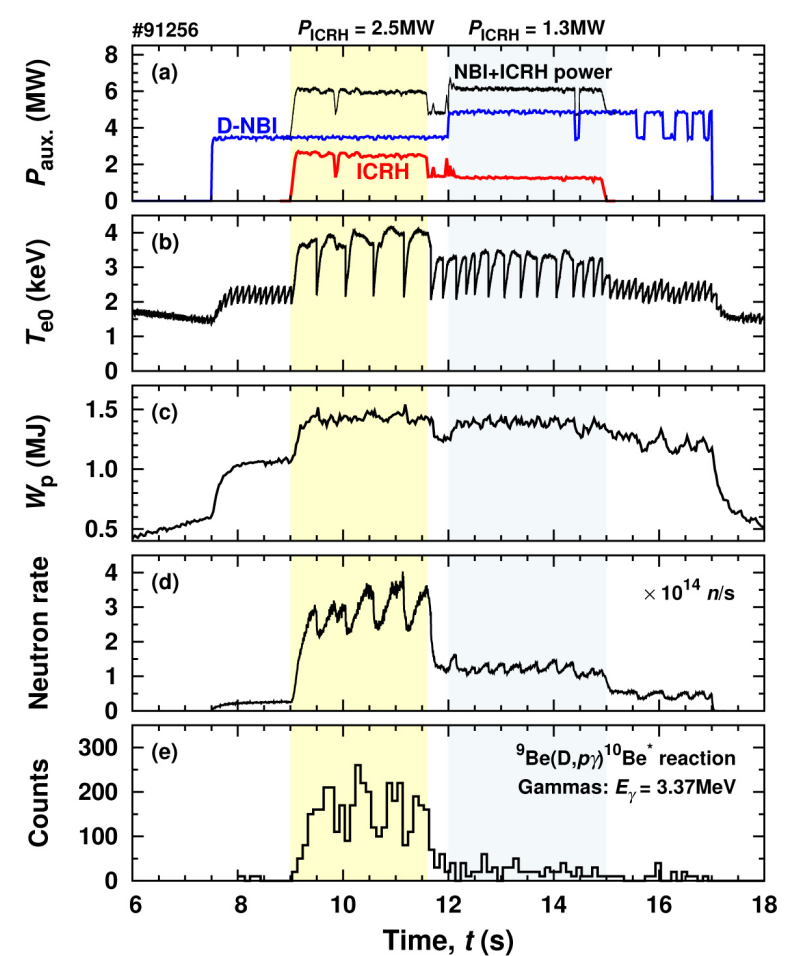

Fig. 3: Evolution of various parameters in JET pulse \#91256 $\left(B_{0} \approx 2.9 \mathrm{~T}, I_{\mathrm{p}}=2.0 \mathrm{MA}, f=25 \mathrm{MHz}\right.$, dipole phasing, $\mathrm{H} /(\mathrm{H}+\mathrm{D}) \approx 0.90)$ : (a) auxiliary heating power (NBI, ICRH and NBI+ICRH); (b) central electron temperature $T_{\mathrm{e} 0}$; (c) plasma stored energy $W_{\mathrm{p}}$; (d) neutron rate; (e) number of gamma-ray counts with $E_{\gamma}=3.37 \mathrm{MeV}$, produced in one of the channels of the D $+{ }^{9} \mathrm{Be}$ nuclear reaction. With $2.5 \mathrm{MW}$ of coupled ICRH power a population of energetic D ions is generated in the plasma as confirmed by the sawtooth stabilization and the increase in the neutron yield and gamma-ray measurements.

The determining role of ICRH for accelerating D particles to high energies is further illustrated in the second phase of the discharge $(t=12-15 \mathrm{~s})$, where the coupled ICRH power was reduced to $1.3 \mathrm{MW}$ and the NBI power was increased to $4.9 \mathrm{MW}$, in order to keep the total heating power approximately constant. This led to a shorter sawtooth period $\left(T_{\text {saw }} \approx 0.25 \mathrm{~s}\right)$ and a reduced number of gamma ray counts. 


\section{Comparison with earlier results obtained in $\mathrm{D}-{ }^{3} \mathrm{He}$ plasmas}

Acceleration of fast $\mathrm{D}$ beam particles in plasma mixtures was already seen before in $\mathrm{D}^{-3} \mathrm{He}$ discharges on TFTR and JET [13-15]. In JET pulse \#55712 the plasma consisted of $\sim 25 \%{ }^{3} \mathrm{He}$ and correspondingly $40-50 \% \mathrm{D}$ [14]. The applied magnetic field was $3.45 \mathrm{~T}$ and the ICRH frequency $f=33 \mathrm{MHz}$. As a result, the distance between the ion cyclotron resonance layers of thermal $\mathrm{D}$ and ${ }^{3} \mathrm{He}$ ions is about $80 \mathrm{~cm}$. The $\mathrm{MC}$ layer in this $\mathrm{D}^{3}{ }^{3} \mathrm{He}$ plasma shifts towards the HFS over about $1.6 \mathrm{~cm}$ for every percent of added ${ }^{3} \mathrm{He}$. A concentration of about $25 \%{ }^{3} \mathrm{He}$ thus locates the MC layer close to the plasma centre, at nearly the same distance of about $40 \mathrm{~cm}$ from both the $\mathrm{D}$ and ${ }^{3} \mathrm{He}$ cyclotron layers.

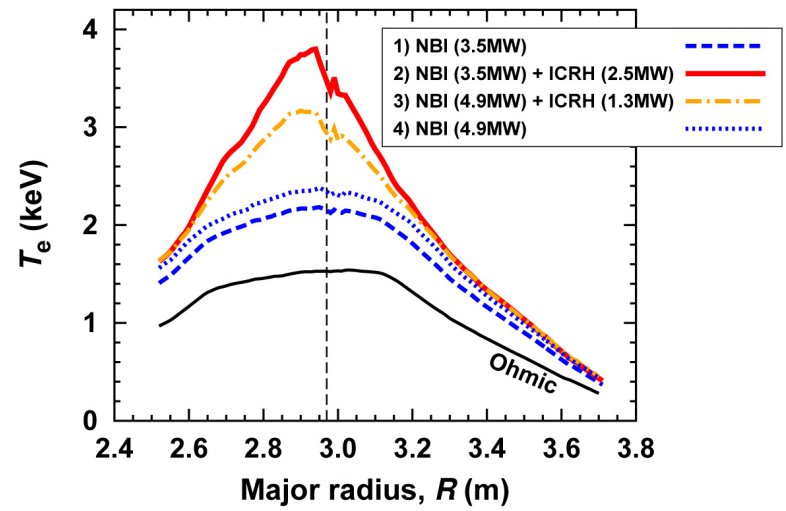

Fig. 4: Electron temperature profiles for JET pulse \#91256 during the ohmic, NBI-only and ICRH+NBI heating phases, averaged over a $1 \mathrm{~s}$ time interval. The dashed vertical line corresponds to the location of the magnetic axis. With $3.5 \mathrm{MW}$ of D-NBI power $T_{\mathrm{e} 0}$ increases from $1.5 \mathrm{keV}$ to $2.2 \mathrm{keV}\left(\Delta \mathrm{T}_{\mathrm{ec}} / \Delta \mathrm{P}_{\mathrm{NBI}} \approx\right.$ $0.2 \mathrm{keV} / \mathrm{MW})$. By adding $2.5 \mathrm{MW}$ of coupled ICRH power, $\mathrm{T}_{\mathrm{e} 0}$ increases further up to $3.8 \mathrm{keV}\left(\Delta \mathrm{T}_{\mathrm{e} 0} / \Delta \mathrm{P}_{\mathrm{ICRH}} \approx\right.$ $0.65 \mathrm{keV} / \mathrm{MW}$ ) and centrally peaked temperature profiles are achieved.

Taking into account the beam injection parameters $\left(E_{0} \approx 120 \mathrm{keV}\right)$ and dominant $k_{\|}$value in the antenna spectrum (dipole phasing) of the earlier experiments, we find that the resonance layer for the fast D-NBI particles is shifted over about $40 \mathrm{~cm}$ towards the LFS. Thus, it coincides with the MC layer, creating the necessary conditions for efficient synergetic acceleration of D-NBI ions with ICRH. That this is indeed the case is illustrated by the strong increase in the neutron yield and long neutron decay times when a blip of D-NBI injection is applied (see Fig. 5, red lines in the bottom panel).

In pulse \#55714, for otherwise similar conditions, ${ }^{3} \mathrm{He}$ ions were replaced by the same amount of ${ }^{4} \mathrm{He}$ ions. As illustrated by the blue dotted line in the bottom panel of Fig. 5, the D- ${ }^{4} \mathrm{He}$ plasma shows a strongly reduced neutron rate and much faster neutron decay. The decay time of the neutron rate in \#55714 is consistent with the slowing down time for $120 \mathrm{keV}$ D-NBI ions [14], indicating no extra acceleration of D-NBI ions with ICRH in D- ${ }^{-4} \mathrm{He}$ plasmas, in contrast to the $\mathrm{D}-{ }^{3} \mathrm{He}$ case (\#55712). Indeed, D and ${ }^{4} \mathrm{He}$ ions have the same $(Z / A)=1 / 2$ and thus there is no MC layer in the plasma and no local amplification of the $E_{+}$field. In fact, pulse \#55714 is very similar to the discharges studied in $[10,11]$, and highlights that fundamental ICRH heating of beam ions can be made significantly much more efficient in plasmas with a purposely chosen particle composition.

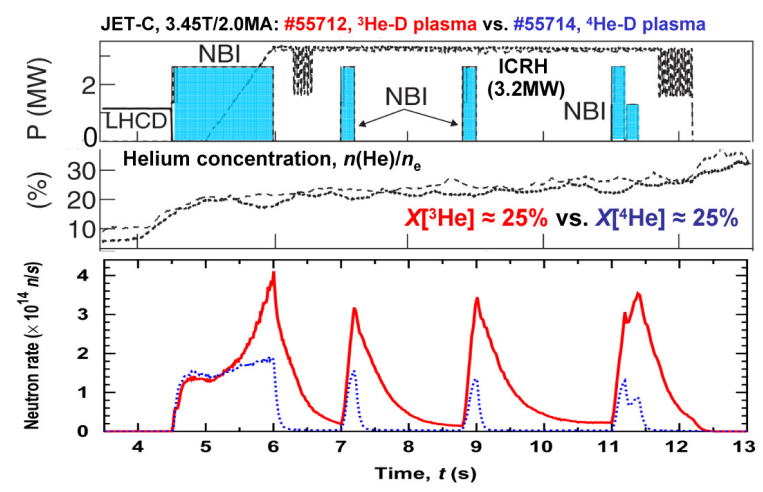

Fig. 5: Time evolution and comparison of various plasma signals from JET pulse \#55712 and \#55714 (adapted from Fig. 21 of Ref. [14]). The plasma in pulse \#55712 consisted of a mixture of D with $\sim 25 \%$ of ${ }^{3} \mathrm{He}$; in pulse $\# 55714{ }^{3} \mathrm{He}$ ions were replaced by ${ }^{4} \mathrm{He}$ for otherwise similar conditions. The presence of a MC layer in pulse \#55712 leads to synergetic acceleration of D-NBI ions to high energies.

This comparison nicely illustrates the key role for the presence of the MC layer and choosing the optimal plasma mix: the same ICRH power absorption mechanism is at work in both the H-D discharges reported here and the $\mathrm{D}-{ }^{3} \mathrm{He}$ discharges discussed in [14].

\section{Relevance for D-T operation}

The insights gained from the experiments reported here leads to a direct extension for use in D-T plasmas. In this section, we briefly discuss how to extend the scenario discussed here towards D-T plasmas in a future DTE2 campaign on JET.

The foreseen parameters of the JET T-NBI system after the recent upgrade are as follows: $\mathrm{E}_{\max }=118 \mathrm{keV}$, power fractions for full-, half- and third-energy components $63 \%, 26 \%, 11 \%$, respectively [16]. This results in an average energy for the injected beam ions $\left\langle\mathrm{E}_{\mathrm{T}-\mathrm{NBI}}\right\rangle \approx 80 \mathrm{keV}$ and an upper estimate for the effective temperature $\mathrm{T}_{\text {eff,T }}=2 / 3<\mathrm{E}_{\mathrm{T}-\mathrm{NBP}}>\approx 53 \mathrm{keV}$ (the average energy and $\mathrm{T}_{\text {eff }}$ for the actual slowing-down distribution of beam ions will be smaller). From Ref. [17] follows that the fusion reactivity in D-T plasmas is maximized at $3 T_{\mathrm{D}}+2 T_{\mathrm{T}} \approx 330 \mathrm{keV}$. Thus for plasma temperatures in the range $T_{\mathrm{i}} \approx 5-15 \mathrm{keV}$, the optimal temperature for 
$\mathrm{T}$ and $\mathrm{D}$ ions to maximize the fusion reactivity should be $\mathrm{T}_{\mathrm{T}} \approx 150 \mathrm{keV}$ and $\mathrm{T}_{\mathrm{D}} \approx 110 \mathrm{keV}$, respectively. This can be realized with appropriate $\mathrm{T}-\left(\mathrm{T}_{\mathrm{NBI}}\right)-\mathrm{D}$ or $\mathrm{T}-\left(\mathrm{D}_{\mathrm{NBI}}\right)-\mathrm{D}$ heating scenarios in D-T plasmas, as explained below.

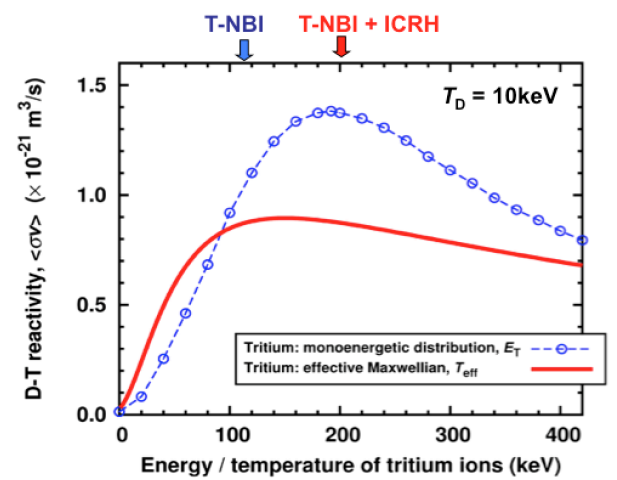

Fig.6: D-T reaction rate $\langle\sigma \mathrm{v}\rangle$ in a Maxwellian D plasma $\left(\mathrm{T}_{\mathrm{D}}=10 \mathrm{keV}\right)$ resulting (i) from the injection of a mono-energetic $\mathrm{T}$ beam (blue dashed line), and (ii) from thermonuclear reactions with a Maxwellian $\mathrm{T}$ ion population with an effective temperature $T_{\text {eff }}>T_{D}$ (red full line).

Figure 6 illustrates D-T $<\sigma$ v $>$ values computed using as an approximation for the accelerated tritium beam particle distribution (i) a mono-energetic $\mathrm{T}_{\mathrm{NBI}}$ beam (using the injection energy as a characterizing parameter) and (ii) a Maxwellian distribution (using the tritium effective temperature $\mathrm{T}_{\text {eff }}$ as characterizing parameter). The maximum fusion yield $<\sigma \mathrm{v}\rangle \approx 1.4 \times 10^{-21} \mathrm{~m}^{3} / \mathrm{s}$ is achieved at $\mathrm{E}_{\mathrm{T}} \approx 200 \mathrm{keV}$ in the first case and $\langle\sigma \mathrm{v}\rangle \approx 0.9 \times 10^{-21} \mathrm{~m}^{3} / \mathrm{s}$ at $\mathrm{T}_{\mathrm{T}} \approx 150$ $\mathrm{keV}$ in the second case. The real optimum $\langle\sigma \mathrm{v}\rangle$ value should be located somewhere between these two extremes.

From Fig. 6 follows that increasing the energy of the injected T-NBI ions by a factor of $\sim 2$ should maximize the fusion output in such plasmas. Moderate acceleration of T-NBI ions can be done in D-T plasmas with T concentrations up to $\sim 30 \%$ at $f=25 \mathrm{MHz}$ and at a toroidal magnetic field $B_{0} \approx 3.7 \mathrm{~T}$ or higher. The advantage of the proposal here compared to earlier tritium minority ICRH experiments in JET [18] is that we now make use of more optimal frequencies for the JET ICRH system $(25 \mathrm{MHz}$ vs. $23 \mathrm{MHz})$ and that the plasma mix is closer to the optimal D-T mixture.

Figure 7 shows 2D TORIC [19] calculations for the ICRF power deposition in a D-T plasma with the following operational parameters in JET-ILW: $B_{0}=3.7 \mathrm{~T}, \quad f=25 \mathrm{MHz}, \quad n_{\mathrm{tor}}=27, \quad n_{\mathrm{e} 0}=6 \times 10^{19} \mathrm{~m}^{-3}$, $T_{0}=5 \mathrm{keV}$. The concentration of the various ions is $X[\mathrm{D}]=68 \%, \quad X[\mathrm{~T}]=30 \%, \quad X\left[\mathrm{~T}_{\mathrm{NBI}}\right]=5 \% \quad$ and $X\left[{ }^{9} \mathrm{Be}\right]=0.5 \%$. The energy distribution of the injected T-NBI ions is approximated by a Maxwellian with an effective temperature $T_{\text {eff }}=55 \mathrm{keV}$. For these conditions, TORIC predicts that most of coupled ICRF power is absorbed by the fast T-NBI ions $\left(p_{\text {abs }}\left(\mathrm{T}_{\mathrm{NBI}}\right)=55 \%\right)$ at $R \approx 2.6 \mathrm{~m}$, in the close vicinity of the MC layer in the D$\mathrm{T}$ plasma.

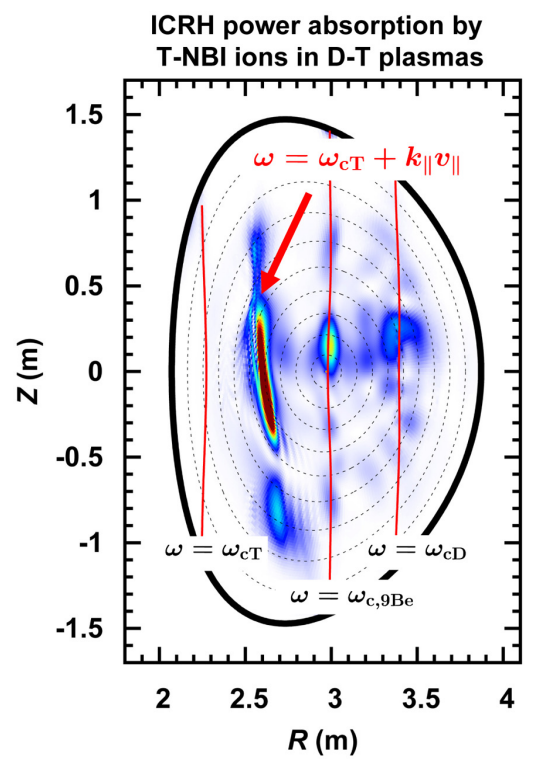

Fig. 7: 2D TORIC computations of the ICRF power absorption for the $\mathrm{T}-\left(\mathrm{T}_{\mathrm{NBI}}\right)$-D three-ion scenario, for a D-T plasma containing $30 \%$ tritium ions. Off-axis absorption of RF power in the vicinity of the MC layer will moderate the acceleration of the T-NBI ions, favourable to maximize the D-T fusion yield.

In contrast to harmonic ICRH absorption scenarios such as $\omega=2 \omega_{\text {ci }}$ and $\omega=3 \omega_{\text {ci }}$ that naturally produce $\mathrm{MeV}$-range energetic ions, the proposed ICRH technique allows a more modest acceleration of the NBI ions in $\mathrm{D}-\mathrm{T}$ plasmas required to maximize the fusion reactivity. Moreover, similar to T-minority experiments reported in [18], off-axis synergetic ICRF acceleration of $\mathrm{T}_{\mathrm{NBI}}$ ions can also be used. This has the additional advantage to reduce the local absorbed RF power density, and is a further possibility to prevent excessive acceleration of the T-NBI ions in order to avoid 'overshooting' the maximum in $\langle\sigma \mathrm{v}\rangle$. As discussed in Ref. [20], additional actuators for controlling fast-ion energies are varying the plasma density $\left(\mathrm{T}_{\text {eff }} \propto 1 / \mathrm{n}_{\mathrm{e}}{ }^{2}\right)$ and concentration of resonant ions (e.g. by varying NBI power). Similarly, DNBI ions can also be used, accelerating them in D-T plasmas through the $\mathrm{T}-\left(\mathrm{D}_{\mathrm{NBI}}\right)-\mathrm{D}$ heating scenario. The proposed ICRF + NBI scenarios offer thus an additional tool to maximize the $Q$-value in the forthcoming DTE2 campaign on JET and are compatible with the existing technological constraints. 


\section{Conclusions}

Absorption of ICRH power by fast D-NBI ions in $\mathrm{H}-\mathrm{D}$ plasmas can be made very effective by arranging the plasma conditions such that the MC layer is located in the close vicinity of the Doppler-shifted resonance layer of the injected fast ions. This is an extension of the three-ion species ICRH technique reported earlier [6-8] to convert traditional electron heating scenarios (through mode conversion) to effective ion absorption scenarios. Synergetic ICRH+NBI acceleration of D-NBI ions was also at work in earlier $\mathrm{D}-{ }^{3} \mathrm{He}$ plasmas [13-15]. A promising extension of this ion cyclotron heating scheme is to accelerate T-NBI and D-NBI ions, which has the potential to further boost the Q-value in future D-T campaigns on JET.

\section{Acknowledgements}

The support from the JET team is warmly acknowledged. This work has been carried out within the framework of the EUROfusion Consortium and has received funding from the Euratom research and training programme 2014-2018 under grant agreement No 633053. The views and opinions expressed herein do not necessarily reflect those of the European Commission and the ITER Organization.

\section{References}

1. ITER Physics Basis, Chapter 6: Plasma auxiliary heating and current drive. Nucl. Fusion, Vol. 39, No. 12, 2496-2539 (1999).

2. Adam, J. Review of tokamak plasma heating by wave damping in the ion cyclotron range of frequency. Plasma Phys. Control. Fusion 29, 443-472 (1987).

3. Porkolab, M. et al.. Recent progress in ICRF physics. Plasma Phys. Control. Fusion 40, A35-A52 (1998).

4. Noterdaeme, J.-M. et al.. Physics studies with the additional heating systems in JET. Fusion Sci. Tech. 53, 1103-1151 (2008).

5. Ongena, J. et al., Recent advances in physics and technology of ion cyclotron resonance heating in view of future fusion reactors. Plasma Phys. Contr. Fusion 59, 054002 (2017).

6. Kazakov, Ye.O. et al, On resonant ICRF absorption in three-ion component plasmas: a new promising tool for fast ion generation. Nucl. Fusion 55, 032001 (2015).

7. Kazakov, Ye.O. et al, A new ion-cyclotron range of frequency scenario for bluk ion heating in deuteriumtritium plasmas: How to utilize intrinsic impurities in our favour. Phys. Plasmas 22, 082511 (2015).

8. Kazakov, Ye.O. et al, Efficient generation of energetic ions in multi-ion plasmas by radiofrequency heating, Nature Physics (2017), advance online publication; http://dx.doi.org/10.1038/nphys4167
9. Kazakov, Ye.O. et al., Study of ICRH scenarios for thermal ion heating in D-T plasmas, Nucl. Fusion 52, 094012 (2012).

10. Krasilnikov, A.V. et al., Fundamental ion cyclotron resonance heating of JET deuterium plasmas, Plasma Phys. Control. Fusion 51, 044005 (2009).

11.Lerche, E.A. et al., Modelling of D majority ICRH at JET: impact of absorption at the Doppler-shifted resonance. Plasma Phys. Control. Fusion 51, 044006 (2009).

12. Kiptily, V.G. et al., Gamma ray diagnostics of high temperature magnetically confined fusion plasmas. Plasma Phys. Contr. Fusion 48, R59-R82 (2006).

13. Darrow, D.S. et al., Enhanced loss of fast ions during mode conversion ion Bernstein wave heating in TFTR, Nucl. Fusion 36, 509-513 (1996).

14. Mantsinen, M.J. et al., Localized bulk electron heating with ICRF mode conversion in the JET tokamak. Nucl. Fusion 44, 33-46 (2004).

15. Van Eester, D. et al., JET $\left({ }^{3} \mathrm{He}\right)-\mathrm{D}$ scenarios relying on RF heating: survey of selected recent experiments. Plasma Phys. Contr. Fusion 51, 044007 (2009).

16. Ciric, D. et al., Performance of upgraded JET neutral beam injectors. Fusion Eng. Design 86, 509-512 (2011).

17. Nath, D. et al., Thermonuclear Fusion Reactivities for Drifting Tri-Maxwellian Ion Velocity Distributions. J Fusion Energy 32, 457-463 (2013).

18. Lamalle, P.U. et al., Expanding the operating space of ICRF on JET with a view to ITER. Nucl. Fusion 46, 391-400 (2006).

19.Bilato, R. et al., Simulations of combined neutral beam injection and ion cyclotron heating with the TORIC-SSFPQL package. Nucl. Fusion 51, 103034 (2011).

20.Stix, T.H., Fast-wave heating of a two-component plasma. Nucl. Fusion 15, 737-754 (1975). 